\title{
The Wrong Type of Pluralism: Toward a Transdisciplinary Social Science
}

Dave Colander, Middlebury College

\author{
Paper prepared for the ICAPE Conference
}

2011 November

\section{MIDDLEBURY ECONOMICS WORKING PAPER}

\section{$11-02$}

\section{DEPARTMENT OF ECONOMICS}

MIDDLEBURY COLLEGE

\section{MIDDLEBURY, VT 05753}

\author{
http://www.middlebury.edu/ econ
}

\begin{abstract}
When heterodox economists talk of pluralism they generally are talking about pluralism within the economics profession - they are asking: how can we have a more pluralistic economics profession? This paper argues that another, perhaps more useful, way to think of pluralism and economics is from the perspective of all the social sciences. When looked in reference to the social science profession rather than in reference to the economics profession, the amount of pluralism increases significantly, since different social sciences follow quite different methodologies. But looking at pluralism from the social science perspective reveals a different type of pluralism problem in social science. While there may be plenty of pluralism within social science as a whole, there is a serious question about whether it is appropriately distributed. This paper argues that heterodox economist's agenda should be a greater blending of all the social science departments. It summarizes proposals to do so on both the undergraduate level and graduate level, and explains why supporting variations of these proposals would be a strategy that would further the objectives of most heterodox economists more so than would their current strategy of pushing for more pluralism in economics.
\end{abstract}

JEL Classifications: A2, B4, B5

Keywords: Pluralism, heterodox, social science, epistemic game theory 


\section{The Wrong Type of Pluralism: Toward a Transdisciplinary Social Science Dave Colander, Middlebury College}

When heterodox economists talk of pluralism (and they are essentially the only economists who talk of it) they generally are talking about pluralism within the economics profession - they are asking: how can we have a more pluralistic economics profession? This paper argues that another, perhaps more useful, way to think of pluralism and economics is from the perspective of all the social sciences. Just as increasing the SIC code from a 4-digit to a 3digit industry increases competition significantly, so too does thinking of pluralism in reference to the social science profession, rather than in reference to the economics profession. When looked in reference to the social science profession rather than in reference to the economics profession, the amount of pluralism increases significantly, since different social sciences follow quite different methodologies. In fact, in reference to the social science profession as a whole, a strong argument can be made that there is too much, not too little, pluralism.

But looking at pluralism from the social science perspective reveals a different type of pluralism problem in social science. While there may be plenty of pluralism within social science as a whole, there is a serious question about whether it is appropriately distributed. The reality is that each social science follows a relatively narrow methodological approach, and there is little conversation and cross fertilization of methods and approaches from one social science to another. So the social science pluralism problem, in my view, is best thought of as a problem of lack of methodological diffusion among social sciences, not a lack of pluralism within any particular social science. The problem is that different methodological approaches are bunched, with sociologists following quite different methodologies than economists, who in turn follow quite different methodologies than behavioral psychologists, anthropologists, and sociologists. In short, there is pluralism in the social sciences, but it is a dysfunctional pluralism. It is as if you poured the flour, milk and eggs into a cake tin, and threw it into an oven without even stirring, let alone blending the ingredients.

The focus of this paper is what to do about it, and my cake metaphor should direct you to my proposed solution. Essentially, my proposed solution is to stir, mix, fold and beat all the social sciences. I argue that heterodox economists should support administratively combining all the social sciences into a single social science department at the graduate level, or at a minimum providing a one year shared core training for all social scientists. At the undergraduate level, I call for a similar blending, or at least the development of a core curriculum of courses that are included in all social science programs. After receiving this core training, students can go off into individual sub fields of social science and specialize.

In other papers (Colander et al. 2009, Colander and Chong 2010) I have outlined proposals that provide that blending on both the undergraduate level and graduate level. In the next part of this paper I briefly summarize those proposals. Then, I explain why I believe supporting variations of these proposals would be a strategy that would further the objectives of most heterodox economists more so than would their current strategy of pushing for more pluralism in economics, with little mention of the other social sciences. 


\section{Unifying Undergraduate Social Science Pedagogy}

In Colander and Chong (2009) I have proposed that all undergraduate students in social science take five core courses that would be developed in conjunction with mathematics, humanities, and philosophy departments. These courses would form a common core of understanding for all social science undergraduate majors, and thereby help diffuse the different approaches used in the different fields. I am not claiming this is sufficient; ideally I would favor combining all the undergraduate social sciences together into a single department, and then letting students specialize after they have a common core. But I see this proposal as a first step towards devising a curriculum devoted to bridging gaps among the social sciences rather than building walls. The five transdisciplinary courses that I propose are the following:

\section{Statistical Foundations of Social Science:}

I start with statistics, because in just about every social science discipline, statistics is already offered. These statistics courses, however, are most often taught in the various departments, rather than in the mathematics departments. This separate teaching of statistics in departments is both costly and divisive_-it doesn't provide a common core of statistical knowledge because each department moves quickly to teaching the statistical approach that dominates in its field. A common core statistics course would let departments who feel more specialized statistical training is needed to offer a follow-up course concentrating on statistical methods used in their field, or to integrate more specialized statistical training into upper-level field courses.

The goal of this common statistics course is (1) to provide all social science students with a background in statistical testing, and its usefulness in evaluating what one can know, and (2) to make all students aware of the limits of statistical testing, and thus be able to identify plausible weaknesses in studies incorporating statistics.

\section{The Mathematics of Cooperation, Coordination, and Conflict:}

This course is essentially an overview of modern game theory. The mathematician, John von Neumann created game theory as the mathematics of the social sciences, and in modern work, it is developing into precisely that. Game theory focuses on the strategic interaction of agents (or decision-making entities). Modern game theory allows a wide range of assumptions about the behavior of individual agents, as well as about the structural context in which they interact. Modern advances in behavioral, evolutionary, and epistemic approaches in game theory have broadened the game theory framework considerably, freeing it from its former preoccupation with high-level rationality and allowing it to incorporate real-world behavioral norms.

Modern game theory thus allows a consideration of norms, culture, and class. It provides a flexible framework for thinking about social problems, without imposing predetermined assumptions about what should happen. As such, it offers a framework and method that transcends any social science, meaning that game theory could help provide a common language and narrative to bridge the gap among the social sciences. 


\section{Complexity and Modeling Foundations of Social Science:}

This would be a mathematical modeling overview course presenting a survey of mathematical models and techniques tailored to the needs of undergraduate social science majors. Its emphasis would be on giving students a sense of the tools used by mathematical social scientists, and the strengths and limits of formal mathematical modeling.

This is not a technical course in the sense of teaching students the techniques of modeling, other than that which is necessary to make its basic concepts concrete. It is more an overview course of mathematical modeling - what a model is, how a model is dependent on its assumptions, and how different assumptions will lead to different conclusions. We see the course distinguishing dynamic models from static models, equilibrium models from non-equilibrium models, multiple equilibria models from unique equilibria models, etc. It would discuss the implications of non-linear dynamics, increasing returns and dynamic feedback in models, and how one can create a model to fit just about any type of observed system behavior.

The goal of the course is to provide students with basic intuition about these different models, and a sense of where different models may be appropriate, or where models may not be appropriate at all, such as in studying what are arguably unique one-off events in history. The course might end with a brief introduction to complexity theory, integrating computational techniques such as agent-based modeling. To reiterate, the goal of the course is not to train students in these techniques, but to give them a consumer's understanding of the techniques. The course would also allow students to identify the techniques that are most relevant to their area of research, and provide a greater vocabulary and foundation for understanding research across disciplines in the future.

\section{Philosophical and Methodological Foundations of Social Science:}

Beyond differences in techniques, fundamental philosophical differences underlie the approaches taken in each of the social sciences, and there is far too little introspection of method in current offerings. This course would be designed to change that. The course would provide students of social science with an understanding of what scholars in different disciplines see as the role of their particular approach in social science.

The course should invigorate students of social science with the usefulness of different approaches in examining different questions. It would discuss social science methodology from a philosopher's perspective, showing the strengths and weaknesses of the methods used in various social sciences. This course would present the multiple roles that social science plays and the different approaches important to each role. We feel that a philosophical approach is particularly suited to such a task. Courses in philosophy already undertake the task of elucidating opposing arguments in these debates without taking sides. This course would only require an orientation towards the needs of the social sciences.

\section{Humanistic Foundations of Social Science:}


The final course I suggest is a course designed to create an awareness of the limitations of an analytical social science approach, and make students better understand the usefulness of humanistic, literary, and historical conceptions of society. Formal modeling of social science will only take one so far, and students should be taught to recognize the limits of social science and formal understanding. English and humanistic history department are populated by scholars who long ago recognized the limitations of formal analysis and concentrated on communicating understanding in more subtle ways.

Understanding these limits is important for all scientists, but it is even more important for social scientists than it is for natural sciences, because social scientists have a different, non scientific, way of understanding social reality. Because social science involves humans studying human interaction, social science offers its practitioners intuitive ways of understanding reality not open to natural scientists. A social scientist can place himself or herself in the place of agent being studied; he can say, "This is what I'd do if I were the agent in the model." An atom studying physics might be able to say that about an atom, but a physicist cannot. That means that there is an alternative path to understanding in social science that does not exist in the natural sciences. Humanists have explored that alternative path to understanding, and have developed a large literature suitable to conveying that understanding, not in the formal way that a natural scientist would, but in an intuitive way that conveys understanding by empathy and shared emotions.

\section{Brief Discussion of the Mix and Content of Courses}

The above courses and their content are offered as suggestions only. I see the actual courses and content of those courses being developed collaboratively by social scientists, philosophers, mathematicians, and humanists, and not by me. Some may object that this set of courses focuses too heavily on models and quantitative techniques, and hence may read our proposal only as an attempt to impose the economist's approach on the other social sciences.

This is emphatically not my goal. I see mathematics as being incorrectly used by many economists, but other social scientists with valid criticisms being unable to challenge them because they don't have the mathematical training to offer alternatives. The mathematical courses I propose are designed to provide social scientists with training to better challenge economic models, and make it difficult for social scientists in general to hide behind mathematics.

The foundational math being suggested here is quite different than the foundational math used in undergraduate economics today. The math I suggest is not a mathematics that presupposes any kind of ideal equilibrium or utilizes only a narrow set of assumptions. Instead, it is math equipped to handle inter-agent disputes and complexity. Rather than simplifying and assuming away the complexities of the real world, the courses are designed to show students how mathematics can be used as a tool to deal with seemingly intractable problems, while also highlighting its limitations. It would show students how quickly simple models can be undermined by processes like feedback loops, increasing returns, learning by doing, non-linear dynamics, and hysteresis, and how norms and culture play an integral role in any social science model. 


\section{Unifying Graduate Social Science Pedagogy}

At the graduate level, I (Colander et al. 2009) have proposed a set of eight courses divided into four modules that would form the core of any social science Ph.D. program. After a first year of taking this core, students could go into studying individual social sciences, or could continue in a unified transdisciplinary social science program that I do not discuss here. I should emphasize that this proposal, as did the undergraduate proposal, concerns the training of social scientists, not of applied social policy specialists. I see the two as quite different, requiring a slightly different mix of methods. Social science training is more mathematical and statistical; applied social policy training is more historical and institutional.

That focus on social science accounts for the emphasis on a mathematical core in both my graduate and undergraduate proposals. In my view, science cannot avoid the quantitative element. It may well be that most undergraduate students should not be studying social science, but rather social policy. In that case, the programs could eliminate much of the mathematical core, or reduce it to a single course which provided students with an overview of what social scientists are doing. It could then concentrate on the philosophical and humanistic transdisciplinary courses relevant for applying the science to policy. However, to the degree that the study is seen as a social science, in my view one cannot escape the mathematical core.

With that caveat, the four modules that I propose are the following.

\section{Module I - Theoretical foundations of human interactions}

Modern Game Theory provides a unified intellectual framework for the study of the social interactions, which especially in its recent developments has proven extremely fruitful. Modern Game Theory is much broader than Classical Game Theory; it includes learning, social norms, endogenous preference and behavioral aspects derived from empirical work.

Nevertheless the major goal of this first module is not to provide some basic knowledge about Modern Game Theory as such, but to make students from various backgrounds understand how it is linked to the broad range of the social sciences in theoretical and conceptual terms, and to provide them with some basic skills to identify real world aspects that can be analyzed in the terms of Modern Game Theory.

\begin{tabular}{|l|l|}
\hline Semester 1 - Classical game theory & Semester 2- Modern game theory \\
\hline $\begin{array}{l}\text { Basic normal form games, equilibrium: } \\
\text { Nash and correlated }\end{array}$ & Social norms \\
Coordination games & Emergence of cooperation \\
Coalitional games & Evolutionary game theory \\
& Epistemic games \\
\hline
\end{tabular}

Module II - Sociometrics - the empirics of social science 
Data is the key link between theory and the underlying reality. As such, understanding the world requires interfacing with data drawn from social settings. We need to link our conceptual ideas with measurable quantities, and to do that, we need a basic idea of how to conceptualize and employ data. The goal of this module is to develop literacy in quantitative empirical methods and an understanding of their limitations, and, finally, the importance of integrating qualitative and quantitative methods to arrive at a final judgment.

\begin{tabular}{|l|l|}
\hline Semester 1 - Data analysis & Semester 2 - Analytic techniques \\
\hline $\begin{array}{l}\text { Tools of pre-theoretical data analysis: } \\
\text { collecting data, descriptive statistics. } \\
\begin{array}{l}\text { Data exploration, data mining and pattern } \\
\text { recognition }\end{array}\end{array}$ & $\begin{array}{l}\text { Multiple regression } \\
\text { Panel methods and time series } \\
\begin{array}{l}\text { Data generation - laboratory, field and } \\
\text { computer experiments }\end{array}\end{array}$ \\
$\begin{array}{l}\text { Integrating qualitative data into analysis } \\
\text { Discrete methods }\end{array}$ \\
\hline
\end{tabular}

\section{Module III - Modeling techniques}

Understanding social questions involves building models to implement our informal understanding and hypotheses. This module is designed to introduce students to a wide range of models and modeling techniques. It introduces them to concepts for aggregate and microscopic modeling. It offers tools for exploring the connection between micro and macro scales, including aggregation and complexity problems. Students can take advantage of some of the elements provided by module I.

\begin{tabular}{|l|l|}
\hline Semester 1 - Modeling dynamics & $\begin{array}{l}\text { Semester } 2 \text { - Modeling agents and their } \\
\text { connections }\end{array}$ \\
\hline $\begin{array}{l}\text { Linear and nonlinear differential and } \\
\text { difference equations }\end{array}$ & $\begin{array}{l}\text { Agent based models } \\
\text { Logic for agent based models } \\
\text { Control of dynamic systems } \\
\text { Deterministic and stochastic systems }\end{array}$ \\
& $\begin{array}{l}\text { Networks } \\
\text { Complexity and emergent properties } \\
\end{array}$ \\
& Modeling uncertainty and risk \\
\hline
\end{tabular}

\section{Module IV-Integrative Approaches}

The current divergence of approaches and thoughts in different branches of the social sciences has historical roots. A student of the social sciences should have an understanding of these 
common roots, of the "big thinkers" whose ideas color current views, and of how and why those ideas became fragmented. The first semester of this module is designed to introduce students to these writings. The second part is to integrate these big ideas with the techniques that have been introduced in the other modules. This second module has two aspects: a creative aspect where students work in groups to do their own modeling and a second forward looking aspect, which introduces the current research frontiers of the various sub-disciplines. We see this module as a transition between the first year core and remainder of the students' studies.

\begin{tabular}{|l|l|}
\hline Semester 1 - History of ideas & Semester 2 - Apps \\
\hline $\begin{array}{l}\text { Epistemological foundations of social } \\
\text { sciences }\end{array}$ & $\begin{array}{l}\text { Group-based projects } \\
\text { Individual-based projects } \\
\text { Classic socioeconomic views - e.g. Smith, } \\
\text { Mill, Marx, Pareto, Durkheim, Weber, } \\
\text { Tocqueville }\end{array}$ \\
$\begin{array}{l}\text { More recent views - e.g. Parsons, Keynes, } \\
\text { Meade, Samuelson }\end{array}$ & $\begin{array}{l}\text { Current frontiers in the specialized social } \\
\text { sciences }\end{array}$ \\
$\begin{array}{l}\text { Bridging natural and social sciences - e.g. } \\
\text { Wilson, Prigogine, Ecological Economics }\end{array}$ & \\
\hline
\end{tabular}

\section{Why Supporting a Social Science Approach to Pluralism Makes Sense for Heterodox Economists}

I fully recognize that the proposals are controversial and stand little chance of being implemented. But even if they have a Don Quixote nature, I would argue that pushing for these proposals are far more likely to advance heterodox economists' pluralist agenda than would an agenda of pushing for more pluralism within economics. Thus, I would argue that it makes sense for heterodox economists to strategically support these proposals as the best way to achieve the more pluralism within economics.

The first reason why is that these proposals ally heterodox economists with professors in psychology, sociology, political science, anthropology, or history, and highlight the strange methodology of mainstream economics. Much of pluralism being called for by heterodox economists is actually simply adding methods and insights being used by other social sciences. By making that explicit, heterodox encomiasts can get administrative support for joint hires, and to put pressure on mainstream economics department to be less methodologically narrow than they currently are.

The second reason is that from an administrator's point of view, the proposals provide a different view of heterodox economists. They shift from being mathophobes who can't make it as serious scientific researchers, as they are often categorized by mainstream economists to 
administrators, to being reasonable economists, who recognize the need to integrate the social sciences, and who are working on the cutting edge of creating a transdisciplinary social science.

To the degree that the reference point can be changed to social science from economics, the rankings of journals changed. Economic journals are narrow field journals. General journals are those related to social science as a whole. In a social science department mainstream economists will have to justify their methodology not only to other economists, but to sociologists, historians, and anthropologists as well, and I suspect more individuals than heterodox economists will be exclaiming that the emperor has no cloths. (Do you really believe that a model that assumes away all heterogeneity for mathematical tractability when you agree that heterogeneity is central to the issue being looked at, is worthy of support for tenure?).

A third reason why this strategy makes sense is that once the models have included the many issues that heterodox economists have called for being included, there is little reason to carve out a separate economics subfield. With the development of evolutionary and epistemic game theory, the core model of modern economics by its very nature goes beyond economic issues and embeds them in social and culture reality. The modern game theory framework is an inclusive social science model that incorporates norms, learning, culture and all aspects of social life. It is still in its rudimentary stages, but there is nothing especially heterodox or mainstream economic about it. It is a general model that can serve as a theoretical foundation for thinking about social problems. Once this model is accepted as the core model of the social sciences, I see no reason to distinguish a heterodox theorist from a mainstream theorist.

A fourth reason why this strategy makes sense for heterodox economists is that it focuses the debate between heterodox and mainstream economics on breath of approaches, not methodological approaches. For example, on the empirical side, there is a widespread acceptance of statistical methods as a way of answering scientific questions by all social scientists. I see no overriding difference between heterodox or mainstream economists or among economists and mainstream social sciences on statistical issues. There is good statistics and bad statistics, and what one wants is the best statistical work one can have, so that you can choose among models. Similarly, with math. The question isn't math; the question is the appropriate math for the issue being looked at. There are legitimate differences among economists and other social scientists about the appropriate statistical procedures to use, but those differences do not, or at least should not, split on heterodox/mainstream lines or along sub discipline lines, except as created by pedagogical hysteresis.

\section{Conclusion}

What I am arguing for may sound radical, but as I have discussed elsewhere, (Colander, 2009, forthcoming) in many ways it is simply returning economics to its social science roots. Classical economists were far more than economists; they were social scientists and social policy specialists. The divisions of social science are a product of the last century; earlier social science had far more overlap. Of course, blending, mixing, beating, and folding are not easy on the batter, and I do not expect any social science to say-yes-let's combine. It is not in their vested interests. How the process of integration will play out will likely differ at different schools, and I would expect that before an acceptable recipe for the baking of the cake is arrived at, there will be lots of lumps and burnt cakes. But with the continual change, vested interests change, and 
eventually I expect that the advantages of a combined social science training will be recognized. Finding the right amount of blending will take time and experimentation. But ultimately, the blending will make for a better cake, and the process of blending will further heterodox economist's agenda more than will advocacy of pluralism within the economics profession.

\section{References}

Colander, David. 2009. “What Was 'It' that Robbins was Defining?” Journal of the History of Economic Thought, 31:4, December, 2009. 437-448.

Colander, David. forthcoming. "Applied Policy, Welfare Economics, and Mill's Half Truths." in The Elgar Companion to Recent Economic Methodology, (John Davis and Wade Hands, editors)

Colander, David and Andrew Chong. 2010. "Increasing Communication among the Socs, the Econs and the Anthros” Middlebury College Working Paper

Colander, David et al. 2009a "Reintegrating the Social Sciences” Report from the Dahlem Conference.

Colander, David, Alan Kirman, Hans Follmer, Brigitte Sloth, Katarina Juselius, Armin Haas, and Thomas Lux. 2009b. “Mathematics, Methods, and Modern Economics” Real-world Economics Review, Issue 50, Sept. 